IdeAs

Idées d'Amériques

15 | 2020

Eau et gestion de l'eau dans les Amériques

\title{
Charlotte de Castelnau-L’Estoile. Páscoa et ses deux maris : une esclave entre Angola, Brésil et Portugal au XVII ${ }^{\mathrm{e}}$ siècle
}

Paris, Presses Universitaires de France, 2019, 303 pages

\section{Silvia Capanema}

\section{OpenEdition}

\section{Journals}

Édition électronique

URL : http://journals.openedition.org/ideas/7967

DOI : 10.4000/ideas.7967

ISSN : 1950-5701

Éditeur

Institut des Amériques

Référence électronique

Silvia Capanema, "Charlotte de Castelnau-L'Estoile. Páscoa et ses deux maris : une esclave entre Angola, Brésil et Portugal au xvıl siècle », IdeAs [En ligne], 15 | 2020, mis en ligne le 01 mars 2020, consulté le 23 septembre 2020. URL : http://journals.openedition.org/ideas/7967 ; DOI : https:// doi.org/10.4000/ideas.7967

Ce document a été généré automatiquement le 23 septembre 2020.

\section{(i) $\ominus$}

IdeAs - Idées d'Amériques est mis à disposition selon les termes de la licence Creative Commons Attribution - Pas d'Utilisation Commerciale - Pas de Modification 4.0 International. 


\section{Charlotte de Castelnau-L'Estoile. Páscoa et ses deux maris : une esclave entre Angola, Brésil et Portugal au XVII ${ }^{\mathrm{e}}$ siècle}

Paris, Presses Universitaires de France, 2019, 303 pages

Silvia Capanema

\section{RÉFÉRENCE}

Charlotte de Castelnau-L'Estoile. Páscoa et ses deux maris : une esclave entre Angola, Brésil et Portugal au XVIIe siècle, Paris, PUF, 303 pages, 2019

1 Faire émerger une vie et la parole d'une femme esclave entre l'Afrique, le Brésil et le Portugal au XvII ${ }^{e}$ siècle, telle est la précieuse contribution de Charlotte de CastelnauL'Estoile. Il s'agit de l'histoire exceptionnelle de Páscoa Vieira, jeune femme née esclave en Angola en 1659 et embarquée pour Salvador de Bahia en 1686 sur un bateau négrier. Entre 1693 et 1703, accusée de bigamie, elle est l'objet d'une enquête de l'Inquisition. Selon l'historienne, c'est aussi un récit de vie exemplaire de la condition des femmes esclaves et de la société esclavagiste dans l'espace atlantique du XvII ${ }^{e}$ siècle. En effet, au moins 300000 âmes arrivent d'Afrique au Brésil entre 1676 et 1700 et pas moins de 175000 entre 1701 et 1725, selon les données du Transatlantic Slave Trade database ${ }^{1}$. Ainsi, entre 1550 et 1856, environ 4,8 millions d'esclaves arrivent au Brésil, ils viennent principalement de deux grandes régions : de la baie du Bénin ou du golfe de Biafra (environ 999600 personnes), et du centre-ouest africain, surtout d'Angola (3,6 millions). Ils arrivent par les ports de Pernambuco, Salvador et Rio de Janeiro, et représentent $45 \%$ du total des Africains déportés vers les Amériques² ${ }^{2}$.

2 Comme c'est le cas de Páscoa, qui avait 27 ans lors de son arrivée au Brésil, nombre de ces Africains deviennent esclaves dans les champs de canne à sucre. À la même époque, 
l'Angleterre importe un nombre équivalent de personnes pour la production du sucre dans ses îles. La particularité de Páscoa est que son histoire est documentée dans les archives de l'Inquisition portugaise, puisque cette femme esclave a été poursuivie pour bigamie, accusée d'avoir épousé Pedro, un esclave au Brésil, alors qu'elle était toujours mariée selon les rites de l'Église catholique en Angola à Aleixo, lui-aussi esclave. Ce procès est exceptionnel par sa durée (10 ans) et sa longueur (114 folios). Un procès long signifie une enquête difficile, des allers-retours et de multiples témoignages et interrogatoires, donc de « la matière » à étudier pour l'historien.

3 Comment Páscoa s'est-elle retrouvée accusée ? Quelle fut sa défense et quelles ont été ses stratégies? Quels ont été le verdict prononcé et sa destinée ? Le lecteur se pose ces questions dès les premières pages, poussé par la curiosité que suscite la vie de cette jeune femme et donc la vie privée des esclaves, leurs mariages, leurs luttes, leurs histoires et les marges de liberté dans les sociétés où ils vivaient. L'ouvrage se place aussi dans la suite de la perspective de l'agency (l'agencement) qui a orienté les approches historiographiques sur l'esclavage dans les dernières décennies, d'autant plus qu'il s'agit de la parole et de l'action d'une femme.

4 L'histoire de la vie de Páscoa est un récit qui peut intéresser les historiens, mais aussi un large public. Il s'agit d'un panorama de la société du xvII e de l'Empire portugais notamment, de Massangano, en Angola, à Salvador de Bahia, au Brésil, en passant par Lisbonne et Castro Marim. C'est l'histoire de l'esclavage et de la traite négrière, mais aussi celle de la circulation de personnes et d'informations dans cet espace Atlantique, celle de la force et de la puissance d'un monde catholique dans lequel l'Inquisition et le mariage représentent deux institutions majeures.

5 Fait exceptionnel, c'est le maître brésilien de Páscoa, le notaire Francisco Álvares Távora né au Portugal, qui la dénonce à l'Inquisition. La seule raison donnée par celuici était sa "crainte de l'Inquisition", ce qui est révélateur du pouvoir de cette institution Outre-Mer, et de celui de l'Église dans la culture et les relations entre maitres et esclaves. L'élément déclencheur de cette dénonciation était la crainte de l'arrivée à Salvador d'un commissaire de l'Inquisition, venu du Portugal pour recevoir les dénonciations. L'auteure suggère d'autres hypothèses: le maitre Francisco Álvares Távora venait d'entreprendre des démarches pour prolonger son droit à conserver son office notarial et il ne voulait pas avoir de problèmes avec l'Inquisition. Peut-être appartenait-il à une famille de nouveaux chrétiens et voulait éviter toute suspicion possible de la part des inquisiteurs? Malgré l'augmentation des prix des esclaves pendant cette période, il préférait "sacrifier» une esclave plutôt que d'avoir des problèmes avec l'Inquisition. Quand son cousin venu d'Afrique lui rapporte que le premier mari de Páscoa était encore vivant et qu'elle serait donc bigame, il décide de la dénoncer et de vendre le mari, Pedro, à un autre propriétaire pour séparer le couple.

6 Páscoa et ses deux maris révèlent les logiques de l'Inquisition. La bigamie était la deuxième cause des procès inquisitoriaux, après le judaïsme (sept cents procès, la grande majorité - $81 \%$ - concernant des hommes).

7 Si les relations commerciales atlantiques sont, comme l'historiographie l'a démontré, directes entre le Brésil et l'Afrique et pas toujours triangulaires pour ce qui est de l'Atlantique sud, une autre forme de triangulation existe concernant le flux d'informations. Le procès de Páscoa révèle en effet que l'enquête était menée aussi bien au Brésil qu'en Angola, en passant par les décisions du Tribunal de l'Inquisition de Lisbonne. 
8 Le récit de la vie de Páscoa, à partir de son procès, suit une perspective chronologique, tout en l'inscrivant dans une problématique plus large en rapport avec le contexte et les dynamiques sociales. En Angola, des relations directes entre le Brésil et l'Afrique étaient mises en place autour du grand marché que représentait la traite d'esclaves, au point que nous pouvons parler d'une "Angola Brasilique" ou d'une Angola qui « se brésilianise » en termes de pratiques culturelles et d'institutions (la production du manioc, base de l'alimentation des esclaves, par exemple). L'esclavage commençait sur le sol africain. Ainsi, Páscoa appartenait à un maitre possédant de nombreux esclaves dans une société complexe, où les statuts des captifs allait des domestiques (mukamas) à des esclaves destinés à la vente (peças), en passant par des travailleurs plus ou moins «libres» (qui ne pouvaient pas être vendus). Páscoa était née esclave et mariée une première fois à Aleixo, lui-même esclave. Toutefois, comme les documents le montrent, Páscoa est vendue et obligée de vivre loin de Massangano parce qu'elle « vivait mal avec son mari » (p.60), le " Nègre Aleixo », selon le témoignage de Miguel da Costa Coito, prêtre à Massangano.

9 Tout permet de penser qu'une fois arrivée au Brésil, Páscoa essaye de créer des liens et ne pas rester isolée, malgré les conditions et le peu de moyens dont elle dispose. Elle se marie avec Pedro, un noir originaire de la côte de Mina, actuel Benin, une année après son arrivée. Plusieurs témoins, des esclaves d'autres propriétaires étaient présents à leur mariage, ce qui permet de croire que le couple possédait des relations et des connaissances. Les esclaves pouvaient se marier sans l'autorisation des maîtres, même si, dans la pratique, ce consentement était important. Dans le cas de Pedro et Páscoa, tous deux acquirent les noms de leur maître ou maîtresse - Álvares pour Pedro et Vieira pour Páscoa. Loin de représenter la liberté, le mariage était toutefois un temps de changement du statut civil des esclaves.

10 Suite à la contre-enquête organisée par Pedro et Páscoa, ils essayent de réagir à leur séparation : face à sa vente, Pedro supplie l'archevêque de Bahia d'intervenir pour que le propriétaire de Páscoa l'autorise à maintenir une vie maritale avec son épouse. Le couple produit des lettres de témoins d'Angola attestant que Páscoa n'est pas mariée en Afrique. Cette correspondance montre que des échanges entre les familles restées en Afrique et les esclaves vendus au Brésil pouvaient exister, ainsi que des actions des esclaves pour changer leur destin et pouvoir vivre en famille. D'autres témoins sont entendus à Salvador et la contre-enquête de Páscoa et son mari produit les effets désirés : le maître admet le retour de Pedro et la reprise de la vie maritale du couple.

11 Toutefois, l'Inquisition n'a pas dit son dernier mot. L'enquête se poursuit, dans le plus grand secret et, à la différence du tribunal ecclésiastique de Bahia, l'Inquisition de Lisbonne est convaincue de la bigamie de Páscoa. Le 12 février 1700, le Tribunal de l'Inquisition ordonne l'arrestation de Páscoa. Le voyage à Lisbonne devait se faire à ses frais, mais comme elle était "pauvre et sans biens ", il est couvert par le capitaine du navire qui espère être remboursé au Portugal. À Lisbonne, dans le cadre de sa défense, elle raconte qu'elle "fut mariée » en Angola avec Aleixo dans un rituel de passage d'anneaux, sans échange de parole et donc, même si elle avait eu une vie maritale avec cet homme et deux enfants, elle n'était pas mariée selon la Sainte Église. Nous apprenons aussi qu'au Brésil elle a eu encore trois enfants avec Pedro dont un avant le mariage, et qu'une fois devenue esclave de Francisco Álvares Távora, elle a eu une relation illicite avec le fils de son maître, Luiz Álvares Távora. Páscoa demande de la miséricorde puisqu'elle « ne savait pas prier le Notre Père ni l'Ave Maria quand elle est 
venue à Bahia» (p. 176). Cette dernière phrase résume l'essence des arguments de défense de Páscoa: elle n'était pas chrétienne avant, et son mariage en Afrique ne fut pas valide pour l'Église. Elle n'est devenue réellement chrétienne que plus tard, et son deuxième mariage fut un vrai mariage. Les juges l'estiment cependant coupable de bigamie, mais sa sentence est atténuée car elle était nouvellement convertie. Elle est condamnée à trois années d'exil dans la forteresse de Castro Martins alors qu'elle a 40 ans.

12 Après deux ans d'exil, Páscoa tente de réduire sa peine en entreprenant des démarches auprès des inquisiteurs. Dans une lettre du 13 mars 1703, elle atteste de sa souffrance dans une terre étrangère, de sa santé faible, et qu'elle souhaite revenir à Bahia pour retrouver la protection et les soins de son maître. Quelques jours plus tard, les inquisiteurs lui accordent la liberté mettant fin à son exil. Après cette dernière pièce du procès, nous perdons la trace de Páscoa. Est-elle retournée au Brésil ? Connaissant la persévérance et la résilience de Páscoa, sa capacité à agir, sa connaissance des procédures et des bons arguments (se montrer une bonne chrétienne et parler de son maître dans sa lettre de demande de remise de peine au lieu de parler de son mari), cela reste de l'ordre du possible.

La belle recherche de Castelnau-L'Estoile permet de comprendre la complexité des relations entre les acteurs historiques, l'action des esclaves dans les marges de leur condition, tout comme le pouvoir de l'Inquisition portugaise qui était un tribunal très bien structuré, mais qui permettait des nuances. Une fois de plus, on comprend la constante mise à jour des formes de domination des maîtres d'esclaves et de leurs réseaux à travers les trois rives de l'Atlantique.

\section{NOTES}

1. https://www.slavevoyages.org/voyage/database

2. ALENCASTRO, Luiz Felipe de, "África, números do tráfico atlântico", In: GOMES, F.; SCHWARCZ, L. M., Dicionário da escravidão e liberdade, São Paulo : Cia das Letras, 2018.

\section{AUTEURS}

\section{SILVIA CAPANEMA}

Silvia Capanema, Docteure en Histoire par l'EHESS, Paris. Maîtresse de Conférences à l'Université

Paris 13- Sorbonne Paris Nord, chercheuse à Pléiade, Campus Condorcet. 